\title{
Revisión del Cuestionario de Emociones Positivas para adolescentes. Propiedades psicométricas de la nueva versión abreviada
}

Review of Positive Emotions Questionnaire for Adolescents. Psychometric properties of the abridged version

LucAs Marcelo Rodriguez ${ }^{\mathrm{a}}$

Centro Interdisciplinario de Investigaciones en

Psicología Matemática y Experimental, Argentina

ORCID: http://orcid.org/0000-0001-5525-1155

María Emilia OÑate

Centro Interdisciplinario de Investigaciones en

Psicología Matemática y Experimental, Argentina

Universidad Católica Argentina, Argentina

Belén Mesurado

Centro Interdisciplinario de Investigaciones en

Psicología Matemática y Experimental, Argentina

\footnotetext{
a Autor de correspondencia. Correo electrónico: lucasmarcelorodriguez@gmail.com

Para citar este artículo: Rodriguez, L. M., Oñate, M. E., \& Mesurado, B. (2017). Revisión del Cuestionario de Emociones Positivas para adolescentes. Propiedades psicométricas de la nueva versión abreviada. Universitas Psychologica, 16(3), 1-13. https://doi.org/10.11144/Javeriana.upsy16-3.rcep
}

\section{RESUMEN}

Las emociones positivas son aquellas experiencias emocionales en las que predomina el placer o bienestar, con una interpretación personal positiva del suceso, por lo que traen consigo una cascada de cambios subjetivos a nivel fisiológico, psicológico, conductual e incluso social. Estas amplían nuestro repertorio de pensamientos y acciones, y potencian la construcción de recursos personales de manera perdurable. El Cuestionario de Emociones Positivas (CEP) para adolescentes mide las emociones de alegría, sentido del humor, optimismo, tranquilidad, gratitud, interés-entusiasmo y satisfacción con la vida. El propósito del Estudio 1 fue evaluar la validez factorial del Cuestionario de Emociones Positivas, Cuestionario de Schmidt (2008) y obtener una versión breve de este; en el Estudio 2 su propuso probar, mediante análisis factorial confirmatorio, la estructura factorial de la versión breve y evaluar la validez de criterio con el comportamiento prosocial. Los resultados muestran una buena validez factorial para el modelo original de 65 elementos con 6 dimensiones. El modelo de 65 ítems se redujo en una versión abreviada de 31 ítems, y se obtuvo un buen ajuste del modelo $\left(\chi^{2} / \mathrm{gl}=1.87 \mathrm{CFI}=0.92 \mathrm{GFI}=0.91\right)$. En cuanto a la valide $z$ de criterio de la versión breve, se encontró que el comportamiento prosocial estuvo asociado con la alegría y el humor $(0.34)$, la gratitud $(0.32)$ y el interés entusiasmo (0.39) dimensiones que componen el Cuestionario de Emociones Positivas. Los resultados se discuten a la luz de las teorías actuales.

Palabras clave

Cuestionario de Emociones Positivas; adolescencia; prosocialidad; análisis psicométrico

\section{ABSTRACT}


Positive emotions are those emotional experiences predominating pleasure or well-being, with a positive personal interpretation of the event, bringing a cascade of subjective physiological, psychological, behavioral changes and even social level. They expand our repertoire of thoughts and actions, and strengthen the construction of personal resources in a lasting way. Positive Emotions Questionnaire (CEP) for adolescents measuring the emotions of joy and humor sense, optimism, peace, gratitude, interest-excitement and satisfaction with life. The purpose the study was, in Study 1, to assess the factorial validity of the Positive Emotions Questionnaire by Schmidt (2008) and get a brief version; the purpose of Studio 2 is to test the factor structure of the brief version by confirmatory factor analysis and evaluate the criterion validity with prosocial behavior. A good factorial validity for the original model of 65 items with 6 dimensions was obtained. The model of 65 items was reduced into a brief version of 31 items, obtaining a good fit model $\left(\chi^{2} / \mathrm{gl}=1.87 \mathrm{CFI}=0.92 \mathrm{GFI}=0.91\right)$. Regarding the criterion validity of brief version we found that the prosocial behavior was associate with joy and humor (0.34), gratitude (0.32) and interest enthusiasm (0.39) dimensions of Positive Emotions Questionnaire. The results are discussed in light of current theories.

Keywords

Positive Emotions Questionnaire; adolescence; prosociality; psychometric analysis

La emoción es uno de los componentes que están presentes en todo proceso psicológico, y uno de los aspectos centrales y más profundos de la experiencia humana (Ortony, Clore, \& Collin, 1990). Las emociones son reacciones complejas que varían en tipo y en intensidad, y que comprometen varios de nuestros sistemas —organismo, cognición y comportamiento-, actuando de manera sincronizada e interrelacionada (Nesse \& Ellsworth, 2009; Scherer, 2005). La experiencia interna de la emoción es sumamente personal y a veces puede resultar hasta confusa, ya que se pueden experimentar varias emociones al mismo tiempo (Plutchik, 2001). A su vez, las emociones y su expresión son cruciales para el desarrollo y la regulación de las relaciones interpersonales (Ekman, 1999).

La amplia gama de emociones puede ser dividida en negativas y positivas. La psicología tradicionalmente ha estudiado las emociones negativas (Fredrickson \& Cohn, 2008) y esto ha sucedido ya que el objetivo de nuestra ciencia ha sido entender y aliviar los problemas psicológicos (Seligman \& Csikszentmihalyi, 2000). Pero también coexiste una tendencia que investiga la forma en la que las emociones enriquecen la experiencia humana y bajo esta intención se encuentra la Psicología Positiva (Seligman, 2002). Algunos autores llamaron emociones positivas a aquellas experiencias emocionales en las que predominaba el placer o bienestar (Lucas, Diener, \& Larsen, 2003), pero Fredrickson (2000) aclara que son mucho más que esto, ya que para que realmente vivenciemos una emoción positiva no alcanza con la agradabilidad sensorial, sino que debe añadirse una interpretación personal positiva del suceso, sea interno o externo; este significado que le demos a la experiencia puede ser consciente o inconsciente y traerá consigo una cascada de cambios subjetivos a nivel fisiológico, psicológico, conductual e incluso social.

Las emociones positivas también difieren de los estados de ánimo positivos, ya que las emociones tienen un objeto específico, suelen ser de corta duración y ocupan el primer plano de la conciencia. En cambio, los estados de ánimo tienen un objeto más difuso, son más duraderos y no están en el centro de nuestra atención consciente (Fredrickson \& Cohn, 2008).

En cuanto a su función, las emociones positivas amplían nuestro repertorio de pensamientos y acciones, y potencian la construcción de recursos personales de manera perdurable (Fredrickson, 1998). Además, aumentan el bienestar subjetivo, la resiliencia, la salud e incluso nos hacen más longevos. También preparan a las personas para tiempos futuros adversos, por ejemplo, la alegría crea la necesidad de jugar, amplía los límites y nos lleva a ser creativos, no solo implica el comportamiento social y físico, sino también el intelectual y el artístico. El desarrollo de estos recursos, como el soporte social, puede ser esencial en momentos de dificultad (Fredrickson, 2001). En otras palabras, las emociones positivas favorecen nuestro crecimiento personal y la conexión social (Vecina Jiménez, 2006). 
La adolescencia media y final es la etapa del ciclo vital donde se van consolidando los desarrollos psicosociales e intelectuales (Arnett, 2008; Bonino, 2005; Griffa \& Moreno, 2005; Piaget \& Inhelder, 2000). Por tal motivo, las emociones positivas cobran una relevancia especial en esta etapa. En esta línea, estudios empíricos actuales (Vega \& Oros, 2013) han demostrado que las emociones positivas empáticas (simpatía y gratitud) pueden potenciar el desarrollo y el ejercicio de conductas socialmente habilidosas en los adolescentes.

Las emociones positivas han sido correlacionadas con diversas variables, entre ellas la prosocialidad. La prosocialidad es el comportamiento de ayuda o beneficio a otras personas, independientemente de las intenciones de esta ayuda (Eisenberg \& Fabes, 1998). Esta puede abordarse de manera global o en contextos específicos (Carlo \& Randall, 2002), pudiendo distinguirse personas mayormente prosociales de las que no lo son; así como evaluar especificidades del constructo, como las tendencias o motivaciones prosociales (Carlo \& Randall, 2002; Richaud, Mesurado, \& Kohan Cortada, 2012; Rodriguez, 2014) o la distinción de los beneficiarios del comportamiento de ayuda, ya sea familiares, amigos o extraños (Mesurado \& Richaud, 2016; Padilla-Walker \& Christensen, 2011).

En nuestro medio, Schmidt (2008) publicó un instrumento para medir la capacidad de experimentar emociones positivas en adolescentes que presentaba buenas propiedades psicométricas. Este contaba con 65 ítems que habían sido determinados teniendo como fundamento entrevistas con adolescentes y tomando en cuenta también el criterio teórico sobre cuáles eran las emociones positivas principales. En la elaboración del Cuestionario de Emociones Positivas de Schmidt (2008) también se consultó a jueces profesionales sobre la pertinencia de los ítems. De esta forma se establecieron seis factores: alegría y sentido del humor, optimismo, tranquilidad, gratitud, interés-entusiasmo y satisfacción con la vida.

En relación con las emociones positivas, McCullough, Kilpatrick, Emmons y Larson
(2001) expresaron que la gratitud promueve la prosocialidad. Dicha afirmación ha sido comprobada en investigaciones empíricas que han destacado el papel de esta emoción positiva como importante facilitador de la prosocialidad (Bartlett \& DeSteno, 2006; Regner, 2009; Tsang, 2006). También se ha afirmado que la alegría generalmente es facilitadora de la conducta prosocial (Salovey \& Rosenhan, 1989). Por otro lado, recientes estudios han mostrado que la serenidad y la satisfacción personal también se encuentran asociadas a las conductas prosociales tanto en varones como en mujeres (Richaud \& Mesurado, 2016). Así mismo, Caprara y Steca (2005) encontraron igualmente una asociación positiva entre la satisfacción con la vida y las conductas prosociales.

$\mathrm{Si}$ bien el Cuestionario de Emociones Positivas (CEP) de Schmidt (2008) tiene buenas propiedades psicométricas, en la práctica resulta algo extenso, sobre todo teniendo en cuenta que en la evaluación psicológica suelen utilizarse diferentes mediciones. Por otro lado, siguiendo las recomendaciones de la Comisión Internacional de Tests (International Tests Commission, 2001) - la cual propone evitar la prolongación innecesaria del tiempo de evaluación-, nos proponemos en este trabajo poner a prueba una versión abreviada del CEP.

Con este propósito se realizaron dos estudios: en el Estudio 1 se va a evaluar la validez factorial del CEP para adolescentes de Schmidt (2008) y obtener una versión abreviada de este; el Estudio 2 se dirigirá con la finalidad de poner a prueba el modelo abreviado obtenido a partir del Estudio 1, mediante el análisis factorial confirmatorio (AFC), así como para evaluar la validez de criterio del modelo abreviado del CEP con una medida de conducta prosocial global. 


\section{Estudio 1}

\author{
Metodología
}

\section{Participantes}

La muestra estuvo compuesta por 280 adolescentes, 103 varones y 177 mujeres, de la provincia de Entre Ríos y Buenos Aires, Argentina. Las edades fluctuaron entre 15 y 19 años, con una media de edad de 16.91 ( $\mathrm{DT}=$ 0.95). La muestra se realizó en instituciones educativas de nivel socioeconómico medio de gestión pública y privada.

\section{Procedimiento}

Se realizaron los acuerdos pertinentes con las instituciones educativas para realizar la toma en horario de dictado de clases. Previamente a la recolección de los datos, se informó a los padres o tutores de los adolescentes, quienes a su vez debían firmar un consentimiento informado autorizando la participación del adolescente. La participación fue voluntaria y anónima. Los protocolos fueron autoadministrables en grupos de 30 alumnos aproximadamente, con la presencia de 2 integrantes del equipo de investigación. En cada caso, la duración fue de aproximadamente 20 minutos.

\section{Instrumentos}

Para evaluar las variables sociodemográficas como edad, sexo, tipo de institución educativa, etc., se elaboró un cuestionario ad hoc. También se solicitó a los participantes que indican el nivel socioeconómico percibido.

Para evaluar las emociones positivas, se utilizó el Cuestionario de Emociones Positivas de Schmidt (2008), el cual consta de 65 ítems y comprende una puntuación tipo Lickert que va desde muy en desacuerdo (1) a muy de acuerdo (4). El cuestionario mide las siguientes emociones positivas:
Alegría y sentido del humor: son dos emociones relacionadas. Por un lado, la alegría como capacidad de reír y sonreír, y, por el otro, el sentido del humor entendido como la capacidad de divertirse a sí mismo y a los demás, contar cosas graciosas, chistes inofensivos, etc. Comprende ítems como: Me considero una persona alegre. La consistencia interna de la subescala en su versión original fue de 0.88 .

Optimismo: emoción caracterizada por esperar que sucedan cosas buenas, mirando siempre el lado positivo de las situaciones y los problemas. Comprende ítems como: Séque las cosas van a salir bien. La consistencia interna de la subescala en su versión original fue de 0.86 .

Tranquilidad: emocionalidad caracterizada por un bienestar profundo que se da al desconectarse de pensamientos inquietantes y se toma distancias de las preocupaciones, estrés, miedo. Comprende ítems como: Aunque la situación sea conflictiva, la mayoría de las veces mantengo la calma. La consistencia interna de la subescala en su versión original fue de 0.87 .

Gratitud: emoción de apreciación por alguien o algo y buen deseo hacia esa persona o cosa, lo que hace surgir las ganas de actuar positivamente. Comprende ítems como: Rara vez me detengo a dar las gracias (puntuación invertida). La consistencia interna de la subescala en su versión original fue de 0.83 .

Interés-entusiasmo: emoción que motiva hacia el cambio y la novedad. Abarca la activación y la orientación para mantener a la persona activamente comprometida con el mundo. Sentimiento de investigar o expandir sí mismo. Comprende ítems como: Me siento motivado para hacer muchas cosas. La consistencia interna de la subescala en su versión original fue de 0.77 .

Satisfacción con la vida: emoción de contentamiento, paz y satisfacción, resultado de la comparación entre querer y necesitar y los logros y habilidades alcanzadas. Se caracteriza por una disposición positiva. Comprende ítems como: En su mayor parte, los recuerdos que tengo sobre mi pasado son negativos (puntuación invertida). La consistencia interna de la subescala en su versión original fue de 0.76 . 


\section{Procedimiento estadístico}

Con el objetivo de estudiar la estructura empírica subyacente del cuestionario, se llevó a cabo a cabo un análisis factorial exploratorio (AFE) de los 65 ítems de escala numérica del CEP, utilizando como paquete estadístico el SPSS 19. Para estudiar la consistencia interna se utilizó el Alfa de Cronbach. Por último, se estudió la correlación entre los factores que componen el instrumento.

\section{Resultados}

El primer objetivo de este trabajo fue evaluar la validez factorial del CEP para adolescentes de Schmidt (2008) en una población argentina de la provincia de Entre Ríos y Buenos Aires. Para evaluar la validez factorial del CEP (Schmidt, 2008), se llevó a cabo un AFE mediante el método de componentes principales y la rotación oblicua (método Oblimin), ya que se consideró que los factores (las diversas emociones positivas) estaban relacionados entre sí. Dicho estudio se realizó en una población total de 280 adolescentes. Previamente se evaluó el índice de adecuación muestral de Kaiser-MeyerOlkin $(\mathrm{KMO}=0.79)$ y la prueba de esfericidad de Bartlett $\left(\chi^{2}=6261.57 ; p=0\right)$, los cuales indicaron que era pertinente realizar el AFE.

Se fijó el número de factores en seis, ya que era lo esperado teóricamente. En la Tabla 1 puede observarse la estructura factorial del CEP. Los seis factores explicaban el $41.48 \%$ de la variancia.

\section{TABLA 1}

Estructura factorial del Cuestionario de Emociones Positivas (CEP)

\begin{tabular}{|c|c|c|c|c|c|c|}
\hline \multirow[t]{2}{*}{$\overline{\text { items }}$} & \multicolumn{6}{|c|}{ Factores } \\
\hline & (a) & (b) & (c) & (d) & (e) & (f) \\
\hline 52. Siempre estoy sonriente & 0.759 & -0.060 & -0.088 & 0.067 & -0.070 & \\
\hline $\begin{array}{l}\text { 30. Me caracterenzo port transm } \\
\text { alegria a los demas }\end{array}$ & 0.740 & 0.015 & 0.133 & 0.062 & -0.039 & 0.086 \\
\hline $\begin{array}{l}\text { 29. Mis compañeros piensan que } \\
\text { soy un persona muy alegre }\end{array}$ & 0.723 & -0.031 & 0.098 & 0.043 & -0.057 & 0.039 \\
\hline $\begin{array}{l}\text { 14. Me caracterizo por mi buena } \\
\text { onda }\end{array}$ & 0.691 & -0.007 & 0.061 & 0.048 & 0.020 & -0.011 \\
\hline $\begin{array}{l}19 \text { Generalmente me rio y } \\
\text { divierto mucho }\end{array}$ & 0.682 & -0.062 & 0.067 & -0.058 & -0.044 & -0.060 \\
\hline $\begin{array}{l}\text { 58. La mayoria de la gente dice } \\
\text { que mi alegria es contagiosa }\end{array}$ & 0.680 & -0.129 & 0.034 & -0.049 & 0.018 & 0.074 \\
\hline $\begin{array}{l}\text { 6. Me considero una persona } \\
\text { alegre }\end{array}$ & 0.635 & -0.090 & 0.110 & 0.228 & -0.002 & -0.096 \\
\hline $\begin{array}{l}\text { 27. Las personas que me } \\
\text { conocen piensan que me falta } \\
\text { sentido del humor" }\end{array}$ & 0.580 & -0.122 & -0.123 & 0.212 & -0.107 & -0.033 \\
\hline $\begin{array}{l}\text { 9. La mayor parte del dia estoy } \\
\text { contento }\end{array}$ & 0.565 & 0.078 & -0.061 & 0.195 & -0.086 & -0.125 \\
\hline $\begin{array}{l}\text { 41. Estoy pendiente de las cosas } \\
\text { graciosas y las digo siempre que } \\
\text { nadie se sienta agrededido }\end{array}$ & 0.495 & 0.012 & 0.017 & -0.128 & -0.171 & 0.029 \\
\hline $\begin{array}{l}\text { 33. Siempre encuentro el lado } \\
\text { gracicoso de las cossas para hacer } \\
\text { reti a los demas }\end{array}$ & 0.492 & 0.041 & 0.020 & -0.186 & 0.114 & -0.251 \\
\hline $\begin{array}{l}60 \text {. Me gystas adicicifarar un poco } \\
\text { los mmomentos serios para hacer }\end{array}$ & 0.422 & -0.013 & -0.015 & -0.116 & 0.225 & -0.052 \\
\hline $\begin{array}{l}\text { reta a los demis } \\
54 \text {. Suelo recme de mi o de orro }\end{array}$ & 0.42 & & & 0.061 & & \\
\hline$\frac{\text { sim ofender a nadie }}{38}$ & & & & & & \\
\hline $\begin{array}{l}\text { 38. Aunque todo vay matl sé } \\
\text { que las cosas van a mejorar }\end{array}$ & -0.046 & -0.072 & 0.124 & -0.164 & -0.006 & -0.721 \\
\hline $\begin{array}{l}\text { 16. Estoy seguro de que el futuro } \\
\text { va a ser mejor }\end{array}$ & -0.088 & -0.055 & 0.038 & -0.086 & 0.088 & -0.704 \\
\hline $\begin{array}{l}\text { 63. Se que las cosas van a salir } \\
\text { bien }\end{array}$ & 0.137 & 0.166 & 0.155 & -0.059 & -0.009 & -0.643 \\
\hline $\begin{array}{l}\text { 13. Las cosas seguirán de mal en } \\
\text { peor" }\end{array}$ & -0.064 & 0.086 & -0.121 & 0.075 & -0.154 & -0.631 \\
\hline $\begin{array}{l}\text { 7. Estoy seguro de que los } \\
\text { problemas se solucionaranan pronto }\end{array}$ & 0.060 & 0.207 & 0.011 & -0.047 & 0.038 & -0.590 \\
\hline $\begin{array}{l}\text { 55. Siempre que me encontre en } \\
\text { appietes supe que todo iba a } \\
\text { estar bien }\end{array}$ & 0.147 & 0.223 & 0.040 & -0.077 & 0.152 & -0.512 \\
\hline $\begin{array}{l}\text { 51. Casi siempre se que las } \\
\text { cosas van a salit bien }\end{array}$ & 0.265 & 0.118 & 0.174 & 0.064 & 0.051 & -0.508 \\
\hline $\begin{array}{l}\text { t0. Siempre se puede rescatar } \\
\text { algo positivo de todas las cosas }\end{array}$ & 0.114 & 0.092 & 0.121 & 0.065 & -0.054 & -0.486 \\
\hline $\begin{array}{l}\text { 34. Pocas veces pienso que el } \\
\text { futurus será mejort" }\end{array}$ & -0.152 & -0.133 & -0.104 & 0.229 & -0.232 & -0.450 \\
\hline $\begin{array}{l}\text { 46. Soy optimista en la mayoria } \\
\text { de las cosas que hago }\end{array}$ & 0.203 & 0.122 & 0.349 & 0.088 & -0.074 & -0.357 \\
\hline 44. Estoy conforme con lo vivido & 0.131 & 0.011 & 0.350 & 0.287 & -0.041 & -0.143 \\
\hline $\begin{array}{l}\text { 12. Puedo encontrar el lado } \\
\text { divertido, aun cuando tengo } \\
\text { mucha tarea escolar }\end{array}$ & 0.543 & 0.111 & 0.051 & -0.049 & 0.082 & 0.008 \\
\hline humor; & $\mathrm{T}$ & & Id & (c) & ter & \\
\hline
\end{tabular}


TABLA 1 (Cont.)

Estructura factorial del Cuestionario de Emociones Positivas (CEP)

\begin{tabular}{|c|c|c|c|c|c|c|}
\hline \multirow[t]{2}{*}{ Items } & \multicolumn{6}{|c|}{ Factores } \\
\hline & (a) & (b) & (c) & (d) & (e) & (f) \\
\hline $\begin{array}{l}\text { 24. Generalmente me manejo } \\
\text { con mucha serenidad }\end{array}$ & 0.069 & 0.770 & 0.123 & -0.062 & 0.000 & 0.096 \\
\hline 56. Me falta tranquilidad* & -0.103 & 0.716 & -0.017 & 0.117 & -0.139 & -0.050 \\
\hline $\begin{array}{l}\text { 32. Aunque la situación sea } \\
\text { confictiva, la mayoria de las } \\
\text { veces mantengo la calma }\end{array}$ & 0.074 & 0.696 & 0.024 & -0.039 & 0.089 & -0.154 \\
\hline $\begin{array}{l}\text { 50. Frecuentemente pierdo la } \\
\text { calma* }\end{array}$ & 0.016 & 0.682 & -0.189 & 0.147 & -0.107 & -0.007 \\
\hline $\begin{array}{l}\text { 4. Soluciono los problemas con } \\
\text { serenidad }\end{array}$ & 0.050 & 0.671 & 0.150 & -0.129 & 0.059 & -0.038 \\
\hline $\begin{array}{l}\text { 18. Me cuesta mantener la } \\
\text { calma }{ }^{*}\end{array}$ & -0.019 & 0.669 & -0.050 & 0.077 & -0.086 & -0.040 \\
\hline $\begin{array}{l}\text { 48. Dicen que transmito } \\
\text { tranquilidad }\end{array}$ & -0.188 & 0.643 & 0.104 & 0.079 & -0.052 & -0.003 \\
\hline $\begin{array}{l}\text { 65. Pierdo la calma hasta por } \\
\text { pequeños inconverientes* }\end{array}$ & 0.011 & 0.637 & -0.136 & 0.101 & -0.144 & -0.075 \\
\hline $\begin{array}{l}\text { 10. Casi nunca me encuentro } \\
\text { tranquilo* }\end{array}$ & -0.199 & 0.627 & -0.017 & 0.059 & 0.105 & -0.136 \\
\hline $\begin{array}{l}\text { 61. Dificimente permanezco } \\
\text { tranqullo* }\end{array}$ & -0.285 & 0.529 & -0.224 & 0.244 & -0.157 & -0.069 \\
\hline 36. Disfruto de la calma & -0.288 & 0.337 & 0.344 & 0.093 & -0.193 & -0.035 \\
\hline $\begin{array}{l}\text { 35. Generalmente olvido } \\
\text { agradecer" }\end{array}$ & 0.049 & 0.195 & -0.021 & -0.105 & -0.691 & 0.137 \\
\hline $\begin{array}{l}\text { 47. Rara vez me detengo a dar } \\
\text { las gracias* }\end{array}$ & 0.039 & 0.139 & 0.015 & -0.060 & -0.654 & 0.048 \\
\hline $\begin{array}{l}\text { 49. Muchas veces soy descortés, } \\
\text { a pesar de haber recibido un } \\
\text { favor* }\end{array}$ & 0.103 & 0.066 & -0.092 & -0.059 & -0.628 & -0.034 \\
\hline $\begin{array}{l}\text { 62. La gente te hace favores por } \\
\text { obligacion o interes. No hay } \\
\text { motivo por el cual agradecert }\end{array}$ & -0.067 & -0.007 & 0.020 & 0.203 & -0.507 & 0.081 \\
\hline $\begin{array}{l}\text { 20. Aprecio las cosas que los } \\
\text { demás hacen por mi }\end{array}$ & 0.251 & 0.065 & 0.126 & -0.177 & -0.467 & -0.085 \\
\hline $\begin{array}{l}\text { 57. Siempre que tengo } \\
\text { oporturidad, devuelvo los favores } \\
\text { recibidos }\end{array}$ & 0.059 & 0.060 & 0.282 & -0.170 & -0.462 & -0.179 \\
\hline $\begin{array}{l}\text { 11. Me cuesta valorar lo que los } \\
\text { demás me brindan* }\end{array}$ & 0.092 & 0.023 & -0.144 & 0.103 & -0.425 & -0.139 \\
\hline $\begin{array}{l}\text { 53. Me interesa muy poco lo que } \\
\text { la gente hace por } \mathrm{mi}^{*}\end{array}$ & -0.077 & -0.175 & 0.003 & 0.075 & -0.409 & -0.009 \\
\hline 45. Disfruto devolviendo favores & -0.015 & 0.190 & 0.553 & -0.101 & -0.337 & -0.020 \\
\hline $\begin{array}{l}\text { 39. Estoy agradecido a varias } \\
\text { personas }\end{array}$ & 0.124 & -0.125 & 0.214 & -0.068 & -0.299 & -0.228 \\
\hline $\begin{array}{l}\text { 64. Existen pocas cosas por las } \\
\text { cuales estar agradecido* }\end{array}$ & -0.214 & 0.011 & 0.162 & 0.337 & -0.297 & -0.100 \\
\hline $\begin{array}{l}\text { 17. Cuando miro a mi alrededor, } \\
\text { encuentro muy pocas cosas por } \\
\text { las que agradecer" }\end{array}$ & -0.092 & -0.185 & 0.083 & 0.492 & -0.157 & -0.221 \\
\hline
\end{tabular}

* Ítem invertido (a) Alegría y sentido del humor; (b) Tranquilidad; (c) Interésentusiasmo; (d) Satisfacción con la vida; (e) Gratitud; (f) Optimismo. Fuente: elaboración propia.
TABLA 1 (Cont.)

Estructura factorial del Cuestionario de Emociones Positivas (CEP)

\begin{tabular}{|c|c|c|c|c|c|c|}
\hline \multirow[t]{2}{*}{ Items } & \multicolumn{6}{|c|}{ Factores } \\
\hline & (a) & (b) & (c) & (d) & (e) & $(\mathrm{f})$ \\
\hline $\begin{array}{l}\text { 8. Siento curiosidad por lo } \\
\text { novedoso }\end{array}$ & -0.054 & -0.077 & 0.573 & 0.102 & 0.089 & -0.004 \\
\hline $\begin{array}{l}\text { 28. Me gusta explorar sobre } \\
\text { diversos temas }\end{array}$ & -0.210 & 0.032 & 0.545 & 0.147 & 0.013 & -0.067 \\
\hline $\begin{array}{l}\text { 31. Me siento motivado para } \\
\text { hacer muchas cosas }\end{array}$ & 0.265 & 0.068 & 0.544 & 0.050 & -0.014 & -0.070 \\
\hline $\begin{array}{l}\text { 42. Me implico fácilmente en las } \\
\text { actividades porque lo disfruto }\end{array}$ & 0.214 & -0.086 & 0.512 & 0.178 & -0.007 & -0.036 \\
\hline $\begin{array}{l}\text { 1. Me intereso por hacer muchas } \\
\text { cosas }\end{array}$ & -0.046 & -0.155 & 0.510 & 0.027 & -0.009 & -0.136 \\
\hline $\begin{array}{l}\text { 43. Siempre trato de obtener la } \\
\text { mayor información posible sobre } \\
\text { aquello que me interesa }\end{array}$ & 0.013 & 0.151 & 0.437 & -0.123 & -0.055 & 0.053 \\
\hline $\begin{array}{l}\text { 2. Todo me entusiasma } \\
\text { 15. Creo haber aprovechado bien }\end{array}$ & 0.263 & -0.201 & 0.331 & -0.074 & 0.033 & -0.065 \\
\hline $\begin{array}{l}\text { las oportunidades que se me } \\
\text { presentaron }\end{array}$ & 0.081 & -0.028 & 0.306 & 0.126 & 0.128 & -0.187 \\
\hline 5. Me arrepiento de varias & & & & & & \\
\hline $\begin{array}{l}\text { decisiones que tomé en el } \\
\text { pasado** }\end{array}$ & -0.064 & 0.093 & 0.109 & 0.623 & 0.127 & 0.160 \\
\hline $\begin{array}{l}\text { 59. Si pudiera volver atrás, haria } \\
\text { las cosas diferentes* }\end{array}$ & -0.023 & 0.028 & 0.136 & 0.606 & -0.034 & 0.125 \\
\hline $\begin{array}{l}\text { 26. Muy pocas veces las cosas } \\
\text { me salieron como yo esperaba }\end{array}$ & 0.031 & 0.157 & -0.006 & 0.563 & 0.010 & -0.127 \\
\hline $\begin{array}{l}\text { 22. Muy pocas veces me siento } \\
\text { realmente contento** }\end{array}$ & 0.354 & -0.071 & -0.053 & 0.561 & -0.171 & -0.066 \\
\hline $\begin{array}{l}\text { 37. En su mayor parte, los } \\
\text { recuerdos que tengo sobre mi } \\
\text { pasado son negativos* }\end{array}$ & 0.102 & 0.115 & 0.267 & 0.493 & 0.006 & 0.132 \\
\hline 25. Me siento derrotado cuando & & & & & & \\
\hline $\begin{array}{l}\text { las cosas no me salen como } \\
\text { deses } 0^{*}\end{array}$ & 0.136 & 0.152 & -0.058 & 0.427 & 0.172 & 0.008 \\
\hline $\begin{array}{l}\text { 21. Me cuesta pensar en } \\
\text { positivo* }\end{array}$ & 0.174 & 0.245 & -0.079 & 0.392 & -0.096 & -0.205 \\
\hline 3. Frecuentemente me pregunt & & & & & & \\
\hline por qué estoy con mala cara* & 0.349 & 0.185 & -0.119 & 0.363 & -0.093 & 0.087 \\
\hline $\begin{array}{l}\text { 23. Raras veces encuentro la } \\
\text { parte divertida de las situaciones } \\
\text { negativas* }\end{array}$ & 0.330 & -0.008 & -0.160 & 0.197 & -0.017 & -0.222 \\
\hline
\end{tabular}

* Ítem invertido (a) Alegría y sentido del humor; (b) Tranquilidad; (c) Interésentusiasmo; (d) Satisfacción con la vida; (e) Gratitud; (f) Optimismo. Fuente: elaboración propia.

En la Tabla 2 se pueden observar las correlaciones $\mathrm{r}$ de Pearson de las subescalas del CEP para adolescentes. 
TABLA 2

Correlaciones $r$ de Pearson de las subescalas

del Cuestionario de Emociones Positivas para

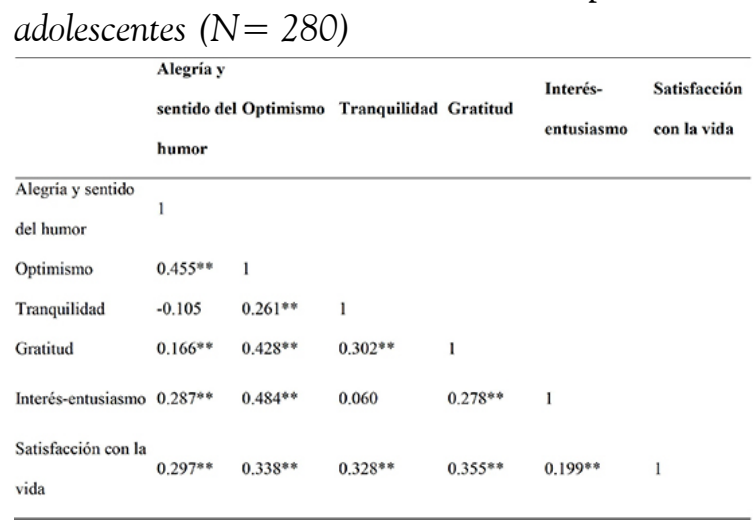

** La correlación es significativa en el nivel 0.01 ( 2 colas)

Fuente: elaboración propia.

Para evaluar la consistencia interna de las dimensiones del CEP se calculó el índice alfa de Cronbach, con los siguientes resultados: alegría y sentido de humor 0.87; optimismo 0.82 ; tranquilidad 0.88 ; gratitud 0.76 ; interésentusiasmo 0.68 , y satisfacción con la vida 0.71 .

Con el propósito de reducir el modelo surgido en el AFE del CEP, para lograr un instrumento de menor extensión, se mantuvieron los ítems que tuviesen una carga factorial elevada. Como resultado se obtuvo un modelo de 31 ítems, a saber: alegría y sentido del humor ítems 6,14 , 19, 29, 30, 52 y 58; optimismo ítems 7, 13, 16, 38 y 63; tranquilidad ítems 4, 18, 24, 32, 50 y 56; gratitud ítems $35,47,49$ y 62 ; interésentusiasmo ítems 1, 8, 28, 31 y 42; satisfacción con la vida ítems 5, 26, 37, 59. El criterio utilizado para la selección de los ítems que se mantuvieron fue la carga factorial de cada ítem en el factor. En las subescalas gratitud, interés-entusiasmo y satisfacción con la vida se mantuvieron los ítems cuya carga factorial fuese mayor a 0.5 ; en las otras tres dimensione (alegría y sentido del humor, optimismo y tranquilidad) el criterio de carga factorial fue más elevado (mayor o igual a $0.68,0.59$ y 0.66 , respectivamente) para lograr un instrumento de no más de 6 ítems por subescalada, teniendo en cuenta la finalidad de realizar una versión abreviada del instrumento. Dicha decisión se tomó teniendo en cuenta los requisitos propios de una estructura simple (Norman \& Streiner, 1996).

\section{Discusión}

En relación al objetivo del Estudio 1: evaluar la validez factorial del CEP para adolescentes de Schmidt (2008), cabe destacar que el AFE pudo llevarse a cabo por el ajuste de los índices $\mathrm{KMO}$ y la prueba de esfericidad de Bartlett, los cuales han sido satisfactorios. El instrumento con sus 6 dimensiones explicó un porcentaje adecuado de la variancia. La matriz de los factores ponderados cumplimentó los siguientes requisitos propios de una estructura simple (Norman \& Streiner, 1996): varianza uniformemente distribuida entre los factores, cada variable cargaba solo en un factor, los pesos de los factores estaban aproximados a 1 o a 0, los factores eran unipolares. Dichos hallazgos muestran una sólida validez factorial del instrumento.

En relación con la confiabilidad, el instrumento mostró tener buenos índices de consistencia interna. Dichos coeficientes son satisfactorios, ya que se consideran buena estimación de consistencia interna alfas entre 0.7 y 0.8 (Kaplan \& Saccuzzo, 2006).

A partir del AFE se redujo el modelo de 65 a 31 ítems a partir de las cargas factoriales, manteniendo aquellos ítems que tuviesen cargas factoriales elevadas (véase el criterio de selección en la sección resultados).

\section{Estudio 2}

\section{Metodología}

\section{Participantes}

La muestra estuvo compuesta por 535 adolescentes, 184 varones y 351 mujeres, de la provincia de Entre Ríos y Buenos Aires, Argentina. Las edades fluctuaron entre 15 y 19 años, con una media de edad de 16.92 (DT= 0.92). La muestra se realizó en instituciones 
educativas de características similares a la del Estudio 1, de nivel socioeconómico medio de gestión pública y privada.

\section{Procedimiento}

Se siguió el mismo procedimiento descrito en el Estudio 1.

\section{Instrumentos}

Para evaluar las variables sociodemográficas como edad, sexo, tipo de institución educativa, etc., se elaboró un cuestionario ad hoc. También se solicitó a los participantes que indican el nivel socioeconómico percibido.

Para evaluar las emociones positivas se usó la versión abreviada resultante del Estudio 1.

Para evaluar la conducta prosocial se utilizó la adaptación a población argentina de la Escala de Prosocialidad de Caprara, Steca, Zelli y Capanna (2005), realizada por Regner y Vignale (2008). Dicha escala mide la conducta prosocial de ayuda, confianza y simpatía en adolescentes y adultos, discriminando sujetos mayormente prosociales de los que no lo son, mediante un puntaje total. Se responde mediante una escala tipo Lickert de 5 opciones que van desde nunca/casi nunca a siempre/casi siempre. Comprende 16 ítems. Ejemplos de ítems: Intento ayudar a los demás; Presto espontáneamente dinero $u$ otras cosas. La consistencia interna del instrumento en nuestra muestra fue de 0.83. Dicho índice es similar a otros estudios argentinos en adolescentes que utilizaron este instrumento, los cuales han informado un alfa de Cronbach de 0.84 (Rodriguez, 2014) y Cronbach de 0.8 (Richaud \& Mesurado, 2016).

\section{Procedimiento estadístico}

Con el objetivo de poner a prueba el modelo abreviado del cuestionario de emociones positivas, se llevó a cabo un análisis factorial confirmatorio (AFC), utilizando el paquete estadístico AMOS .17. Para estudiar la consistencia interna se utilizó el Alfa de Cronbach. Por último, para estudiar la validez de criterio se estudió la relación entre los factores que componen el CEP abreviado y la conducta prosocial.

\section{Resultados}

Con el propósito de poner a prueba el modelo abreviado de 31 ítems del CEP, se llevó a cabo un AFC con el método de estimación de parámetros por máxima verosimilitud. Dicho estudio se realizó en una muestra de 535 adolescentes. Para considerar el ajuste del modelo, se tuvieron en cuenta el índice comparativo de ajuste (CFI) y el índice de bondad de ajuste (GFI). Se calcularon como medidas del error el Root Mean Square Residual (RMR) y el promedio de los residuales estandarizados al cuadrado (RMSEA).

Los resultados del modelo de 31 ítems con 6 dimensiones fueron los siguientes: $\chi^{2}(419)=$ 782.79, $\mathrm{p}=0, \chi^{2} / \mathrm{gl}=1.87, \mathrm{CFI}=0.92, \mathrm{GFI}=$ $0.91, \mathrm{RMR}=0.035$ y RMSEA $=0.04$. Dichos resultados indicaron un buen ajuste del modelo propuesto. En la Figura 1 se puede observar el modelo de medición y las cargas factoriales estandarizadas del CEP revisado de 31 ítems.

Para evaluar la consistencia interna de las dimensiones del CEP en su versión de 31 ítems se calcularon los índices alfa de Cronbach, con los siguientes resultados: Alegría y sentido de humor 0.88; Optimismo 0.72; tranquilidad 0.85; gratitud 0.68; interés-entusiasmo 0.68 , y satisfacción con la vida 0.65 .

Para evaluar la validez de criterio del CEP revisado se utilizó la muestra de 535 adolescentes. Se realizaron correlaciones $\mathrm{r}$ de Pearson con la Escala de Conducta Prosocial. La conducta prosocial correlacionó positivamente con las 6 emociones positivas: Alegría y sentido del humor (0.337), optimismo (0.281), tranquilidad (0.144), gratitud $(0.320)$, interés-entusiasmo (0.391) y satisfacción con la vida (0.142). Todas las correlaciones fueron significativas al nivel 0.01 . 
Figura 1.

Modelo de medición y cargas factoriales estandarizadas del Cuestionario de Emociones Positivas revisado de 31 ítems

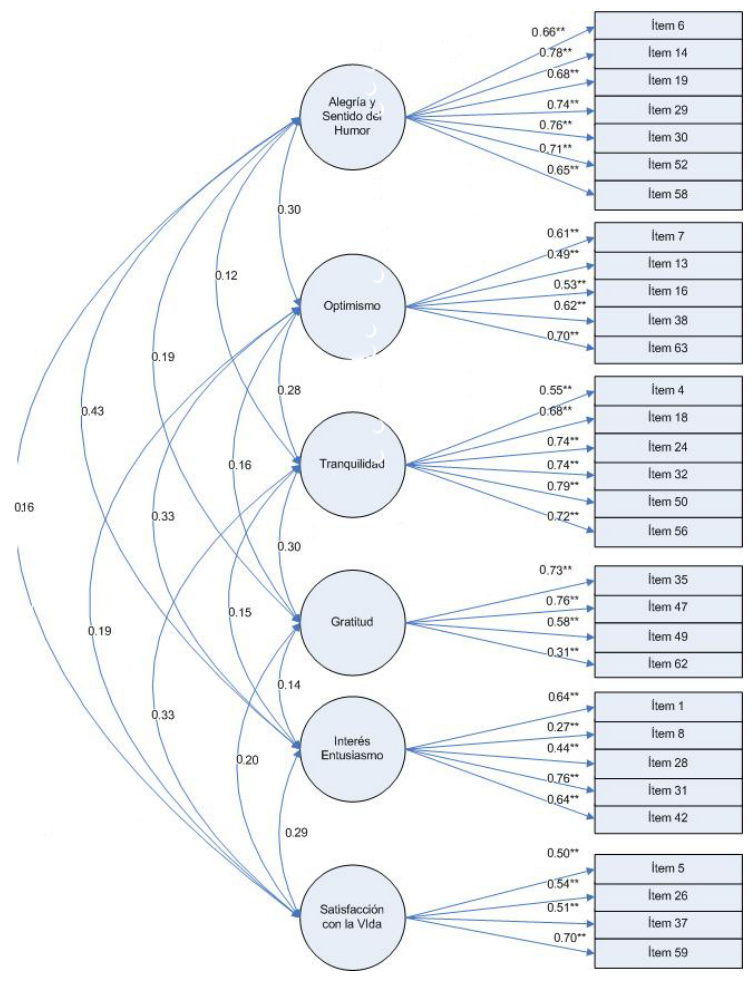

Fuente: elaboración propia.

\section{Discusión}

En relación con el objetivo del Estudio 2: poner a prueba mediante el AFC el modelo abreviado, se realizó el AFC del modelo de 31 ítems.

Se recomienda utilizar múltiples indicadores para evaluar el ajuste del modelo. Ejemplo de esto es el uso del chi cuadrado y este estadístico sobre los grados de libertad $\left(\chi^{2} / g l\right)$, debido a que el chi cuadrado es sensible al tamaño de la muestra, también el índice de ajuste comparativo (CFI) y el índice de bondad de ajuste (GFI). Un $\chi^{2} / g l$ inferior a 2 indica un buen ajuste del modelo. Así mismo, CFI y GFI superiores a 0.9 indican un ajuste satisfactorio entre el modelo teórico y los datos empíricos (Cupani, 2012).

El AFC del modelo de 31 ítems dio como resultado un buen ajuste, ya que el $\chi^{2} / g l$ fue inferior a 2 (1.87). Los demás índices $(\mathrm{CFI}=$
0.91 y GFI = 0.92) señalan un ajuste satisfactorio entre el modelo teórico y los datos empíricos, ya que ambos fueron superiores a 0.9 (Cupani, 2012). El índice de error RMSEA se considera óptimo cuando es inferior a 0.06 ( $\mathrm{Hu} \&$ Bentler, 1998), lo cual ocurrió en el modelo de 31 ítems.

En relación con la consistencia interna, en el modelo de 31 ítems los índices alfa de Cronbach fluctuaron entre 0.65 y 0.88 . Dichos coeficientes son satisfactorios, ya que se consideran buena estimación de consistencia interna alfas entre 0.70 y 0.80 (Kaplan \& Saccuzzo, 2006). Inclusive escalas con escaso número de ítems y buena evidencia de validez y razones prácticas o teóricas pueden estar alrededor de 0.60 (Loewenthal, 2001).

En el Estudio 2 además se buscaba evaluar la validez de criterio del nuevo modelo propuesto (modelo de 31 ítems), a través de su correlación con la Escala de Conducta Prosocial (Caprara et al., 2005). Se encontraron las mayores correlaciones con alegría y sentido del humor (0.337), gratitud (0.320) e interés entusiasmo (391). Estos resultados son coincidentes con los estudios que evidencian la gratitud como variable facilitadora y relacionada con la prosocialidad (Bartlett \& DeSteno, 2006; McCullough et al., 2001; Regner, 2009; Tsang, 2006), así como las teorizaciones que vinculan la alegría como facilitadora de la prosocialidad (Salovey \& Rosenhan, 1989). Cabe destacar que no se han encontrado antecedentes de estudios que vinculen el interés y entusiasmo con la conducta prosocial. Podría pensarse que el interés y entusiasmo, entendido como la activación y la orientación para mantener a la persona activamente comprometida con el mundo (Schimidt, 2008), estaría vinculado con la ayuda a otras personas independientemente de la intencionalidad que se persiga (Eisenberg $\&$ Fabes, 1998), por el hecho de estar activamente comprometido con el mundo y el entorno, lo que predispondría a la persona a estar más atenta a las oportunidades de ayuda a otros. 


\section{Conclusiones generales}

La presente investigación es un aporte importante para la evaluación psicológica en un tema central de la psicología positiva como lo son las emociones positivas.

Los resultados obtenidos han permitido plantear un nuevo modelo de 31 ítems, conservando las 6 dimensiones propuestas por el autor original: alegría y sentido del humor, optimismo, tranquilidad, gratitud, interés entusiasmo y satisfacción con la vida (Schmidt, 2008). El Cuestionario de Emociones Positivas revisado para adolescentes muestra sólidas propiedades psicométricas con buena validez de constructo. De aquí que este trabajo contribuya a la comunidad científica, aportando un instrumento breve de evaluación validado en una muestra hispanoparlante, lo que suele ser de utilidad para los profesionales e investigadores hispanos.

Para estudiar la validez de criterio en este artículo, nos centramos en analizar las relaciones entre las emociones positivas y la conducta prosocial como una conducta global. Nuestros resultados fueron en la misma dirección de investigaciones previas, las cuales mostraron una relación moderada entre los distintos tipos de emociones positivas y la conducta prosocial (Richaud \& Mesurado, 2016). Es probable que la alegría y el sentido del humor, como la capacidad de reírse de sí mismo y de reír o divertirse con los demás, así como una actitud optimista de la vida y el entusiasmo, contribuyen a que ese tono emocional positivo nos predisponga a realizar favores a los demás o a tener conductas de ayuda hacia ellos. Como mostraron también las investigaciones previas, el sentimiento de gratitud es un factor importante asociado con las conductas prosociales, ya que este sentimiento de valoración de las acciones de los otros predispone a actuar positivamente hacia los demás (Bartlett $\&$ DeSteno, 2006).

Así mismo, es conocida la frase pronunciada por Crandall y Putnam en los años 80. Ellos afirmaban que si uno quiere conocer las características de personalidad que tienen un importante efecto sobre la capacidad de encontrar satisfacción en la vida, el "primer candidato" es el interés social. Ese interés social muchas veces se manifiesta con acciones o conductas concretas de ayuda que explican esa relación con la satisfacción con la vida, consistentes en varios estudios (Caprara \& Steca, 2005; Weinstein \& Ryan, 2010).

Por último, en relación con la tranquilidad, también se encontró una leve relación con las conductas prosociales en adolescentes, tal como muestran investigaciones previas en niños (Cuello \& Oros, 2014). Estas autoras sugieren que altos niveles de serenidad facilitan el desarrollo de las conductas prosociales en los chicos, manifestándose en acciones como confortar a alguien que está triste, ayudar a un compañero de clase a hacer la tarea, compartir dulces con otros, acompañar a sus amigos, etc. Esto puede deberse a que la serenidad amplía el alcance de la atención, lo que los lleva a poder descubrir las necesidades de los otros (Cuello \& Oros, 2014; Fredrickson \& Branigan, 2005).

\section{Limitaciones y futuros estudios}

Cabe destacar entre las limitaciones del presente estudio que sus variables han sido íntegramente evaluadas con una modalidad autoadministrable; además, la muestra ha sido intencional, lo que podría generar un sesgo en el muestreo, aunque se hayan tomado los recaudos pertinentes. Además, es importante señalar que por las características de la muestra (intencional, no aleatoria y de solo dos regiones de Argentina) se deben tomar precaución para la generalización de los presentes datos.

En relación con futuras líneas de investigación, debería ampliarse la muestra, no solo en número, sino también en representatividad de la población. Además, deberían seguir evaluándose las propiedades psicométricas de la nueva versión del instrumento, así como su validez de criterio con otras variables como la emocionalidad positiva y negativa. Podría incluirse la evaluación de otras emociones positivas que no están contempladas en el cuestionario, y continuar trabajando en la consolidación de instrumentos 
válidos y confiables para la medición de constructos de la psicología positiva en nuestro medio.

\section{Referencias}

Arnett, J. J. (2008). Adolescencia y adultez emergente. Un enfoque cultural. México: Pearson.

Bartlett, M. Y., \& DeSteno, D. (2006). Gratitude and prosocial behavior. Helping when it costs you. Psychological Science, 17(4), 319-325.

Bonino, S. (2005). Contagio, empatía e comportamento prosociale. En G. V. Capraray, \& S. Bonino (Eds.), Il comportamento prosociale. Aspetti individuali, familiari e sociali (pp. 23-44). Trento: Erikson.

Caprara, G. V., \& Steca, P. (2005). Selfefficacy beliefs as determinants of prosocial behavior conducive to life satisfaction across ages. Journal of Social and Clinical Psychology, 24(2), 191-217. http:// dx.doi.org/10.1521/jscp.24.2.191.62271

Caprara, G., Steca, P., Zelli, A., \& Capanna, C. (2005). A new scale for measuring adults' prosocialness. European Journal of Psychological Assessment, 21 (2), 77-89.

Carlo, G., \& Randall, B. (2002). The development of a measure of prosocial behaviors for late adolescents. Journal of Youth and Adolescence, 31, 31-44.

Cuello, M., \& Oros, L. (2014). Serenity and its relationship to prosocial and aggressive behaviors in Argentinean children. Journal of Latino/Latin American Studies, 6(1), 31-39.

Cupani, M. (2012). Análisis de ecuaciones estructurales: conceptos, etapas de desarrollo y un modelo de aplicación. Revista Tesis, 1, 186-199.

Crandall, J. E., \& Putnam, E. L. (1980). Relations between measures of social interest and psychological well-being. Journal of Individual Psychology, 36, 156-168.
Eisenberg, N., \& Fabes, R. (1998). Prosocial development. En W. Damon (Ed. de la serie), \& N. Eisenberg (Ed. Vol.), Handbook of Child Psychology. Vol. 3. Social, Emotional, And Personality Development (5. ${ }^{\circ}$ ed., pp. 701-778). New York: John Wiley.

Ekman, P. (1999). Basic emotions. En T. Dalgleish, \& M. J. Power (Eds.), Handbook of Cognition and Emotion. Chichester: Wiley.

Fredrickson, B. L. (1998). What good are positive emotions? Review of General Psychology, 2, 300-319. http:// dx.doi.org/1089-2680.2.3.300

Fredrickson, B. L. (2000). Cultivating positive emotions to optimize health and well-being. Prevention 8 Treatment, 3(1), 1a.

Fredrickson, B. L. (2001). The role of positive emotions in positive psychology. The broaden-and-build theory of positive emotions. American Psychologist, 56(3), 218-226. http:// dx.doi.org/10.1037//0003. O66X.56.3.218

Fredrickson, B. L., \& Branigan, C. (2005). Positive emotions broaden the scope of attention and thoughtactions repertoires. Cognition and Emotion, 19, 313-332. http:// dx.doi.org/10.1080/02699930441000238

Fredrickson, B. L., \& Cohn, M. A. (2008). Positive emotions. En M. Lewis, J. Haviland, \& L. F. Barrett (Eds.), Handbook of emotions (3rd ed.). New York: Guilford Press.

Griffa, M. C., \& Moreno J. E. (2005). Claves para una psicología del desarrollo. Tomo 2. Buenos Aires: Lugar Editorial.

Hu, L. T., \& Bentler, P. M. (1998). Fit indices in covariance structure modeling: Sensitivity to under parameterized model misspecification. Psychological Methods, 3, 424-453.

International Tests Commission (2001). International guidelines for test use. International Journal of Testing, 1 (2), 93-114.

Kaplan, R. M., \& Saccuzzo, D. P. (2006). Pruebas psicológicas: principios, aplicaciones y temas. 6. $\circ$ ed. Madrid: International Thomson. 
Loewenthal, K. M. (2001). An introduction to psychological tests and scales. 2. ${ }^{\circ}$ ed. Londres: Psychology Press.

Lucas, R. E., Diener, E., \& Larsen, R. J. (2003). Measuring positive emotions. En Lopez, S. J., \& Snyder, C. R. (Eds.), Positive Psychological Assessment: A Handbook of Models and Measures. Washington: American Psychological Association. http://dx.doi.org/10.1037/10612-000

Mesurado, B., \& Richaud, M. C. (2016). The relationship between parental variables, empathy and prosocial-flow with prosocial behavior toward strangers, friends, and family. Journal of Happiness Studies, 1-18.

McCullough, M. E., Kilpatrick, S.D., Emmons, R. A., \& Larson, D. B. (2001). Is gratitude a moral affect? Psychological Bulletin, 127(2), 249-266.

Nesse, R. M., \& Ellsworth, P. C. (2009). Evolution, emotions, and emotional disorders. American Psychologist, 64(2), 129-139. http://dx.doi.org/10.1037/ a0013503

Norman, G. R., \& Streiner, D. L. (1996). Bioestadística. Madrid: Mosby.

Ortony, A., Clore, G. L., \& Collin, A. (1990). The cognitive structure of emotion. Cambridge: Cambridge University Press.

Padilla-Walker, L. M., \& Christensen, K. J. (2011). Empathy and self-regulation as mediators between parenting and adolescents' prosocial behavior toward strangers, friends, and family. Journal of Research on Adolescence, 21 (3), 545-551.

Piaget, J., \& Inhelder, B. (2000). Psicología del niño. $15^{\circ}$ Ed. Madrid: Morata.

Plutchik, R. (2001). The nature of emotions. American Scientist, 89, 344-350.

Regner, E., \& Vignale, P. (2008). Adaptación de la Escala de Conductas Prosociales de Caprara y Pastorelli. Manuscrito no publicado.

Regner, E. (2009). Compasión y gratitud, emociones empáticas que elicitan las conductas prosociales. En M. C. Richaud, \& J. E. Moreno (Eds), Investigación en Ciencias del Comportamiento. Avances
Iberoamericanos. Buenos Aires: Ediciones CIIPME-Conicet.

Richaud, M. C., \& Mesurado, B. (2016). Las emociones positivas y la empatía como promotores de las conductas prosociales e inhibidores de las conductas agresivas. Acción Psicológica, 13(2), 31-42. http:// dx.doi.org/10.5944/ap.13.2.17808

Richaud, M., Mesurado, B., \& Kohan Cortada, A. (2012). Analysis of dimensions of prosocial behavior in an Argentinean sample of children. Psychological Reports: Mental $\mathbb{E}$ Physical Health, 111(3), 1-10.

Rodriguez, L. M. (2014). Motivaciones y conductas prosociales en adolescentes argentinos. Praxis. Revista de Psicología, 16(25), 79-87.

Salovey, P., \& Rosenhan, D. L. (1989). Mood states and prosocial behavior. Handbook of Social Psychophysiology (371-391). Oxford: John Wiley \& Sons.

Scherer, K. R. (2005). What are emotions? And how can they be measured?. Social Science Information, 44(4), 695-729. http:// dx.doi.org/10.1177/0539018405058216

Schmidt, C. M. (2008). Construcción de un cuestionario de emociones positivas en población entrerriana. Revista Iberoamericana de Diagnóstico y Evaluación Psicológica, 26(2),117-139.

Seligman, M. (2002). La auténtica felicidad. Argentina: Ediciones Zeta.

Seligman, M., \& Csikszentmihalyi, M. (2000). Positive psychology: An introduction. American Psychologist, 55, 5-14. http:// dx.doi.org/10.1037/0003-066X.55.1.5

Tsang, J. A. (2006). Gratitude and prosocial behaviour: An experimental test of gratitude. Cognition and Emotion, 20(1), 138-148. http:// dx.doi.org/10.1080/02699930500172341

Vega, N. G. De la, \& Oros, L. B. (2013). El rol de las emociones positivas empáticas en el comportamiento social de adolescentes argentinos. Psicodebate, 13, 9-23.

Vecina Jiménez, M. L. (2006). Emociones positivas. Papeles del Psicólogo, 27(1), 9-17. 
Weinstein, N., \& Ryan, R. M. (2010). When helping helps: Autonomous motivation for prosocial behavior and its influence on well-being for the helper and recipient. Journal of Personality and Social Psychology, 98(2), 222-244. http://dx.doi.org/10.1037/ a0016984

\section{Anexo}

\section{Cuestionario de Emociones Positivas (CEP), versión revisada}

Marque en cada oración con una cruz la alternativa que corresponde a su caso

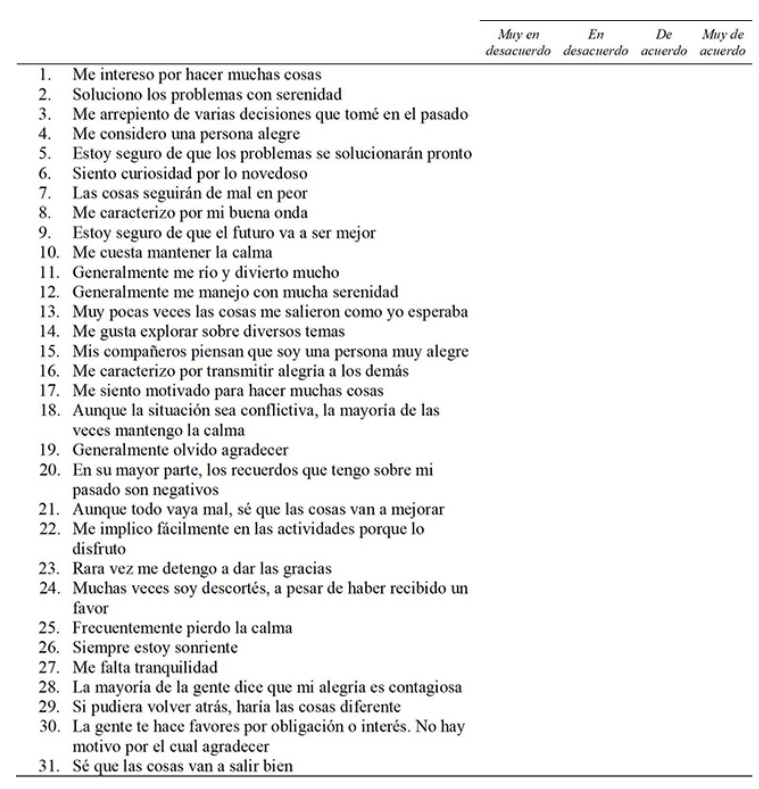

\section{Notas}

* Artículo de Investigación. 